\title{
Anabolizantes esteroideos y ginecomastia. Revisión de la literatura
}

\author{
D. A. DE LUIS**, R. ALLER**, L. A. CUÉLLAR**, C. TERROBA**, \\ E. ROMERO*,**
}

Sección de Endocrinología y Nutrición Clínica. Hospital Universitario del Río Hortega. *Hospital Clínico Universitario. **Instituto de Endocrinología y Nutrición Clínica (IEN). Facultad de Medicina. Valladolid

\section{RESUMEN}

La ginecomastia es una situación común consistente en la proliferación del componente glandular de la mama masculina como resultado de un disbalance en la acción de estrógenos y andrógenos a nivel del tejido glandular. La etiología es muy variable, destaca en el momento actual los anabolizantes esteroideos. Los estudios realizados analizando este problema en pacientes que toman anabolizantes son escasos aunque la prevalencia de ginecomastia entre estos pacientes es de hasta un 52\%. Presentamos un caso clínico donde se muestra el desarrollo en pocos meses de ginecomastia bilateral asimétrica secundaria a la toma de anabolizantes, con respuesta parcial al tamoxifen. Por tanto ante un paciente con hábitos deportivos que presente ginecomastia, una de nuestras primeras sospechas debe ser la toma de anabolizantes. Tras la suspensión de los anabolizantes y la instauración de un ciclo de tratamiento oral preferentemente con tamoxifen, la tasa de remisiones puede ser alta en función del tiempo de duración de la ginecomastia y del tamaño. Si no se consigue la remisión la cirugía es una buena opción.

PALABRAS CLAVE: Esteroides anabolizantes. Ginecomastia
ANABOLIC STEROID AND GYNECOMASTIA. REVIEW OF LITERA TURE

\section{ABSTRACT}

Gynaecomastia is a common situation, with a proliferation of glan dular component of male breast secondary to an imbalance in sexual hormones in mammary tissue. A main cause of gynaecomastia is anabo lic steroides consumption. Few studies have been performed in patients with high consumption of anabolic steroids, showing a high prevalence $(52 \%)$. In this article we present a case of bilateral gynaecomastia secondary to anabolic steroids intake, with a partial response to tamoxi fen. In summary, a patient with sportive habits and gynaecomastia, an early suspicious diagnosis is anabolic steroid intake. After suppression of anabolic steroid intake and treatment with tamoxifen, a high remission rate can be achieved. If gynaecomastia persisted, surgery is a good option.

KEY WORDS: Anabolic steroids. Gynaecomastia.

De Luis DA, Aller R, Cuellar LA, Terroba C, Villar A, Romero E. Anabolizantes esteroideos y ginecomastia. Revisión de la literatura. An Med Interna (Madrid) 2001; 18: 489-491.

\section{INTRODUCCIÓN}

La ginecomastia es una situación común consistente en la proliferación del componente glandular de la mama masculina como resultado de un disbalance en la acción de estrógenos y andrógenos a nivel del tejido glandular. En general, todo tejido mamario visible o palpable en el varón debe considerarse anormal excepto en tres situaciones fisiológicas como son las edades; perinatal, puberal y senil. La incidencia de este problema varía según las series dependiendo sobre todo del tamaño del disco mamario que se tome como partida y de la edad del grupo.
La etiología es muy variable, habiéndose implicado diversos problemas como; tumores, endocrinopatías, hipogonadismo primario y secundario, fármacos, drogas, etc. Dentro del grupo de los fármacos destacan en el momento actual los anabolizantes esteroideos. Los estudios realizados analizando este problema en pacientes que toman anabolizantes son escasos aunque la prevalencia de ginecomastia entre estos pacientes es de hasta un $52 \%$ (1).

Presentamos un caso clínico donde se muestra el desarrollo en pocos meses de ginecomastia bilateral asimétrica secundaria a la toma de anabolizantes, con respuesta parcial al tamoxifén.

Trabajo aceptado: 19 de enero de 2001

Correspondencia: D.A. de Luis. Prof Asociado Endocrinología. Director Ejecutivo IEN. C/ Caamaño, 51 Bis $3^{\circ}$ C. 47013 Valladolid. 


\section{CASO APORTADO}

Presentamos el caso de un varón de 19 años que practicaba culturismo. Acudió a la consulta con su madre, al detectar esta el aumento del tamaño de ambas mamas, desde hacía 6 meses.

El paciente no presentaba ningún antecedente médico-quirúrgico de interés. Había iniciado un programa intensivo de entrenamiento para competir en los Campeonatos Nacionales, con un programa de levantamiento de pesas y una dieta hiperproteica. La exploración física general fue normal (Tensión arterial 120/75 mmHg) con ginecomastia bilateral de predominio derecho, sin galactorrea ni secreción sanguinolenta en los pezones a la presión $(5 \mathrm{~cm}$ de tejido mamario derecho y $3 \mathrm{~cm}$ de tejido mamario izquierdo). En la analítica inicial la bioquímica general fue normal (función hepática y renal normal), el hemograma fue normal y la ecografía mamaria mostró tejido mamario sin componente graso.

El perfil hormonal inicial mostró valores normales de T4l, TSH, IGFI, estradiol, PRL y beta hCG. Se detectó unos niveles de testosterona libre $48 \mathrm{pg} / \mathrm{ml}$ (valores normales en varones $80-280 \mathrm{pg} / \mathrm{ml}$ ), FSH 2 UI/l (valores normales 1-10UI/l), LH 3 UI/l (valores normales 1-9 UI/l), mostrando por tanto un patrón de hipogonadismo hipogonadotropo.

En el interrogatorio dirigido el paciente refería disminución de la libido y negó el consumo de marihuana, opiaceos, neurolépticos, alcohol u otros fármacos vía oral relacionados con la ginecomastia. No obstante refirió el uso desde hacía un año de inyecciones intramusculares de estanazolol, nandrolona, alternado con ciclos orales de danazol y mesterolona. Desde hacía 1 mes, no había tomado ninguna de estas medicaciones. Inciamos tratamiento con $10 \mathrm{mg}$ cada 12 horas vía oral de tamoxifen durante 6 meses, remitiendo la ginecomastia en la mama izquierda y no así en la mama derecha. Se realizó tratamiento quirúrgico de esta última.

\section{DISCUSIÓN}

Las causas de ginecomastia son múltiples (2), a continuación enumeramos las más significativas; asociada a tumores (productores de estrógenos o precursores, como son los tumores testiculares de células de Leydig, suprarrenales o incluso productores de hCG como el coriocarcinoma), ginecomastia familiar, hipogonadismo hipergonadotrópico (síndrome de Klinefelter, defectos enzimáticos de la síntesis de testosterona, hermafroditismos y alteraciones testiculares adquiridas como radiación, traumatismo o infecciones), hipogonadismo hipogonadotrópico, ginecomastia idiopática, fármacos (Tabla I) y otras causas o misceláneas (cirrosis, tirotoxicosis, realimentación, infección por VIH, estress psicológico, lesión espinal, etc.).

En el grupo de fármacos (Tabla I) que producen ginecomastia los anabolizantes esteroideos están ocupando cada vez un apartado más importante, al presentar gran importancia el culto al cuerpo en nuestra sociedad (3). La identificación de un fármaco responsable de la ginecomastia es importante porqué podemos evitar costosos estudios diagnósticos, al suspender el fármaco la ginecomastia es con mucha frecuencia reversible y además otros efectos secundarios relacionados con la función reproductiva como el hipogonadismo que presentaba nuestro paciente pueden también estar presentes y ser evitados al retirar el fármaco.

Los estudios realizados entre culturistas y atletas de élite son cada vez más numerosos $(1,4-7)$. Mostrando como estos pacientes se administran tanto vía oral como parenteral dosis medias de anabolizantes de 250-3.200 mg por semana, en ciclos que oscilan entre 4 y 12 semanas (4). Alrededor de un

\section{TABLA I}

FÁRM ACO S Y DRO GAS PRO DUCTO RES DE GINECO M ASTIA

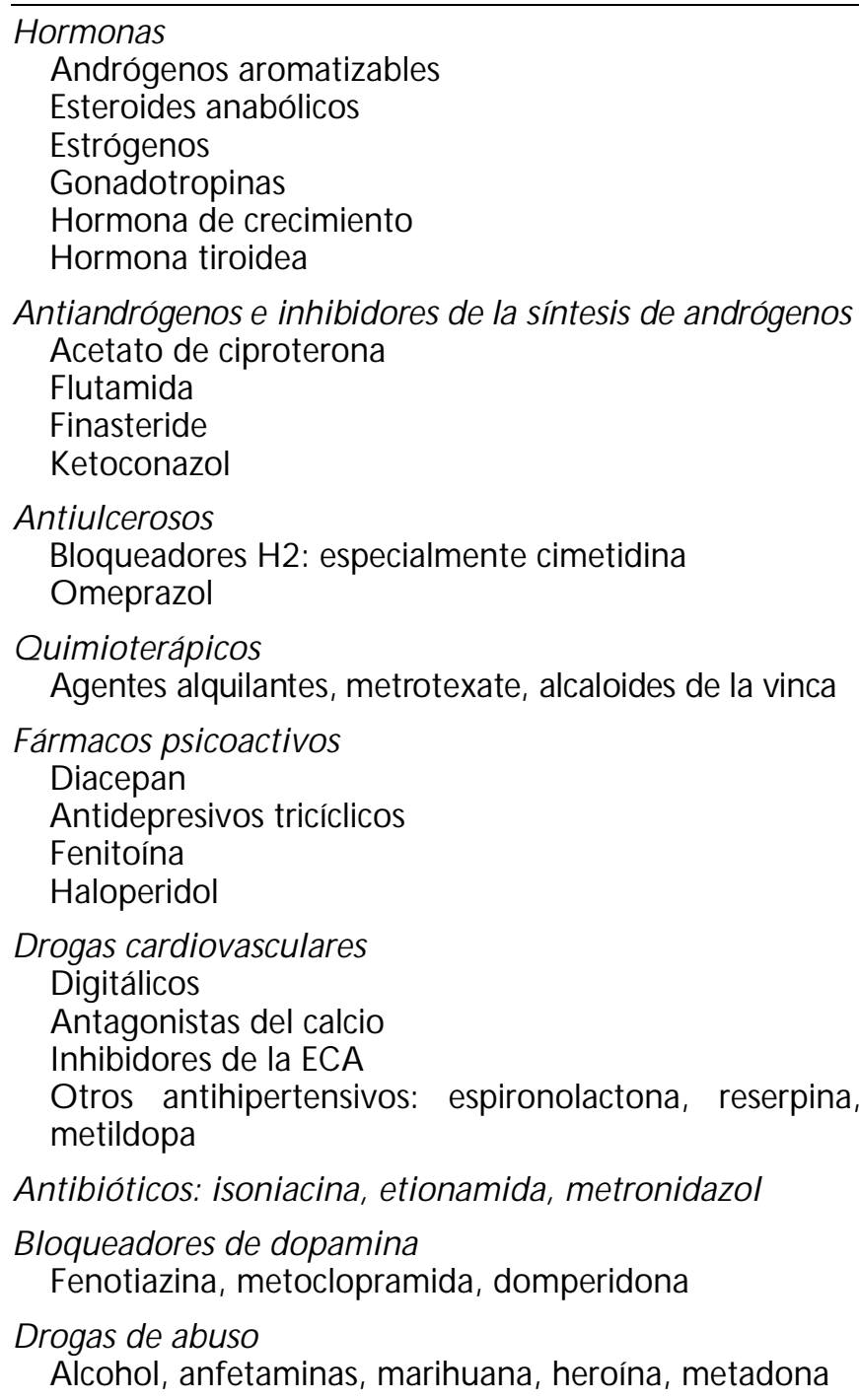

$9,1 \%$ de los varones y $2,3 \%$ de las mujeres encuestadas entre atletas de élite han tomado en alguna ocasión anabolizantes (1). El patrón habitual de consumo es a través de entrenadores o de amigos que practican el mismo deporte con una tasa de auto-administración elevada, presentando efectos secundarios muy diversos. Los efectos secundarios encontrados son un $56 \%$ atrofia testicular, 52\% ginecomastia, 36\% hipertensión, $56 \%$ retención de líquidos y $26 \%$ lesiones tendinosas. Entre las alteraciones analíticas destacan, modificaciones en las transaminasas y en la creatinina, así como un patrón de hipogonadismo hipogonadotropo.

Ante cualquier paciente donde se diagnostique ginecomastia, si se identifica una patología de base responsable debe ser tratada y si el aumento de las mamas es de reciente comienzo lo más probable es que remita. El mecanismo que puede explicar la ginecomastia secundaria a fármacos en nuestro paciente es el disbalance entre los niveles de andrógenos y estrógenos a nivel mamario; por una disminución en los niveles de andrógenos, incremento en los niveles de estrógenos, 
incremento de la sensibilidad del tejido mamario y/o defectos en los receptores de andrógenos. En los pacientes donde la ginecomastia parece tener relación con algún fármaco este debe suspenderse como en el caso de anabolizantes o sustancias tóxicas o ser reemplazado por otro si el fármaco es necesario. En algunas situaciones es necesario utilizar tratamiento farmacológico. Se han utilizado tres tipos de terapias en los pacientes con ginecomastia; andrógenos, antiestrógenos e inhibidores de la aromatasa.

Con respecto a los andrógenos, la testosterona no es útil salvo en los pacientes con hipogonadismo, pudiendo ser aromatizada a estradiol agravando el problema. La dehidrotestosterona no sufre aromatización por lo que mantiene su capacidad para inhibir el crecimiento mamario. El danazol, un derivado de la 17 alfa etinil testosterona también se ha utilizado produciendo una mejoría limitada en el $23 \%$ de los pacientes (8).

Los dos antiestrógenos más estudiados son el citrato de clomifeno y el tamoxifeno. Con clomifeno (9) un 64\% de los pacientes consiguen una remisión completan de su ginecomastia. Los efectos secundarios (náuseas, alteraciones visuales y rash) que aparecen cuando se utiliza para inducir ovulación no suelen aparecer cuando se usa en el tratamiento de la ginecomastia, probablemente porque las dosis son menores (50-100 mg/día o $50 \mathrm{mg}$ a días alternos). El tamoxifén (10) en dosis de $10 \mathrm{mg}$ cada 12 horas aproximadamente en el $80 \%$ de los pacientes tratados experimentan una reducción parcial o total de la ginecomastia en los tres primeros meses de tratamiento. En general no aparecen efectos secundarios cuando se administra más de 4 meses salvo algunos episodios de nauseas y molestias epigástricas.

La testolactona (11) inhibe la actividad aromatasa bloqueando la transformación de andrógenos testiculares y supra- rrenales en estrógenos. A dosis de $150 \mathrm{mg}$ cada 8 horas no inhibe la secreción de gonadotropinas ni retrasa el desarrollo puberal teniendo hasta un $40 \%$ de efectividad.

Como norma general en pacientes con ginecomastia dolorosa o de reciente comienzo es recomendable un ciclo de tratamiento con tamoxifen durante tres meses. También puede usarse en pacientes con ginecomastia de larga duración aunque en pocos casos se conseguirá una remisión completa debido a la fibrosis, como fue en parte nuestro caso. En situaciones en las que el tratamiento médico es inefectivo o inapropiado es necesaria una solución definitiva sobre todo en individuos jóvenes o con macroginecomastia. La reconstrución mamaria (mamoplastia) puede dar un resultado excelente. La liposucción no elimina el tejido glandular pero puede disminuir llamativamente el tamaño de la mama al quitar tejido graso y muchos casos pueden ser tratados con liposucción solo o en combinación con la excisión quirúrgica directa. Se aconseja respetar una pequeña parte del tejido glandular subaerolar para evitar deformidades posteriores. En nuestro caso se realizó cirugía en una de las mamas al no responder al tratamiento farmacológico, hay que tener en cuenta en nuestro caso la ginecomastia era de 6 meses de evolución y con un diámetro superior a los $4 \mathrm{~cm}$ en la mama derecha. Ya se han descrito casos en la literatura de tratamiento quirúrgico en la ginecomastia secundaria a anabolizantes con buenos resultados (12).

En resumen ante un paciente con hábitos deportivos que presente ginecomastia, una de nuestras primeras sospechas debe ser la toma de anabolizantes. Tras la suspensión de los anabolizantes y la instauración de un ciclo de tratamiento oral preferentemente con tamoxifén, la tasa de remisiones puede ser alta en función del tiempo de duración de la ginecomastia y del tamaño. Si no se consigue la remisión la cirugía es una buena opción.

\section{Bibliografía}

1. Korkia P, Stimson GV. Indications of prevalence, practice and effects of anabolic steroid use in Great Britain. Int J Sports Med 1997; 18 (7): 557-62.

2. Courtiss EH. Gynecomastia: An analisis of 159 patients and current recommendations for treatment. Plast Reconstr Surg 1987; 79: 740-53.

3. Porcerelli JH, Sandler BA. Anabolic-androgenic steroid abuse and psychopathology. Psychiatr Clin North Am 1998; 21: 829-833.

4. Evans NA. Gym and tonic: a profile of 100 male steroid users. Br J Sports Med 1997; 31: 54-58.

5. Neild D. Gynecomastia in bodybuilders. Br J Clin Pract 1995; 49: 172

6. Pope HG, Katz DL. Psychiatric and medical effects of anabolic-androgenic steroid use. A controlled study of 160 athletes. Arch Gen Psychiatry 1994; 51: 375-82.

7. Strauss RH, Yesalis CE. Anabolic steroids in the athlete. Annu Rev Med 1991; 42: 449-57.
8. Jones DJ, Holt SD, Surtees P, Davison DJ, Copcoat MJ. A comparison of danazol and placebo in the treatment of adult idiopathic gynaecomastia: result of a prospective study in 55 patients. Ann R Coll Surg Engl 1990; 72: 296-298.

9. LeRoith D, Sobel R, Glick SM. The effect of clomiphene citrate on puberal gynecomastia. Acta Endocrinol 1980; 95: 177-80.

10. Parker LN, Gray DR, Lai MK, Levin R. Treatment of gynecomastia with tamoxifen a double-blind crossover study. Metabolism 1986: 35: 705-708.

11. Zachmann M, Finholzer U, Muritano M. Treatment of puberal gynecomastia with testolactone. Acta Endocrinol Suppl 1986; 279: 218-226.

12. Reyes RJ, Zicchi S, Hamed H, Chaudary MA, Fentiman IS. Surgical correction of gynecomastia in bodybuilders. Br J Clin Pract 1995; 49: 177-179. 